\title{
Interface Property and Apparent Strength of High-Strength HydRophilic FibER IN CEMENT MATRIX
}

\author{
By Tetsushi Kanda ${ }^{1}$ and Victor $\mathrm{C} . \mathrm{Li}^{2}{ }^{2}$ Member, ASCE
}

\begin{abstract}
This study addresses the characterization of fiber-matrix interfacial properties and the apparent strength of high-strength hydrophilic fibers. Single-fiber pullout bond tests and single-fiber pull-to-rupture strength tests were conducted by employing polyvinyl alcohol (PVA) fibers. The pullout bond tests showed that these fibers have surprisingly high chemical and frictional bond strengths. The chemical bond strength was relatively stable independent of a water-to-cement ratio of matrix and the fiber type tested, contrary to the friction bond strength. The pull-to-rupture strength tests revealed that the apparent strength of the PVA fibers in cementitious composites is considerably lower than that in standard fiber strength tests. The apparent strength was further reduced with inclining angle of fiber alignment. This effect was captured by a simple phenomenological model in this study, which introduces the apparent strength reduction factor. The combined effects of high bond strength and degraded fiber strength will likely contribute to composite performance less than would be expected from a high-performance fiber.
\end{abstract}

\section{INTRODUCTION}

Flexible fibers with a hydrophilic nature have been developed with high tensile strength. Representative fibers include polyvinyl alcohol (PVA) fibers and aramid fibers. These fibers typically have a diameter of $10-20 \mu \mathrm{m}$ and a tensile strength of 2,000-2,500 $\mathrm{MPa}$. They are expected to show a strong bond with cementitious matrix due to their hydrophilic nature, even though little research has been conducted to quantitatively evaluate their interfacial properties. Furthermore, the cost of the PVA fibers is even lower than steel fibers on an equal volume basis. Because of such promising characteristics, many researchers have attempted to achieve high performance in random short fiber reinforced cementitious composites (RSFRCC) with these hydrophilic fibers (Akihama et al. 1985; Betterman et al. 1995; Maalej et al. 1995). However, the performance of those composites did not seem to attain a level as high as expected. For example, PVA-RSFRCC was found to show lower tensile strength than a carbon fiber composite, although the PVA fibers used for the tests have twice the tensile strength of carbon fibers (Akihama et al. 1982, 1985). Both composites had almost the same mix proportion with only a difference in fiber type. This example illustrates that careful consideration is necessary in composite design to fully utilize the performance of high-strength hydrophilic fibers.

Despite the need for such consideration, comprehensive methodology has not yet been established for RSFRCC designed with hydrophilic fibers. For this purpose, two outstanding issues need to be resolved: 1) Characterization of interfacial property; and 2) characterization of fiber strength in a cementitious matrix. The first issue, interfacial characterization, involves direct pullout tests with single fiber embedded in matrix (Li and Chan 1995). However, these tests are not easy to perform with high-strength hydrophilic fibers since the fibers tend to rupture rather than pull out completely due to their small diameter and high bond strength. In this study, this problem is overcome by substantially reducing the embedment length of fibers in specimens employing a microfiber testing

\footnotetext{
'Grad. Student, ACE-MRL, Dept. of Civ. and Envir. Engrg., Univ. of Michigan, Ann Arbor, MI 48109-2125. (On leave from Kajima Corporation).

${ }^{2}$ Prof. and Dir., ACE-MRL, Dept. of Civ. and Envir. Engrg., Univ. of Michigan, Ann Arbor, MI.

Note. Associate Editor: Nemkumar Banthis. Discussion open until July 1,1998 . To extend the closing date one month, a written request must be filed with the ASCE Manager of Journals. The manuscript for this paper was submitted for review and possible publication on June 24, 1996. This paper is part of the Journal of Materials in Civil Engineering, Vol. 10, No. 1, February, 1998. CASCE, ISSN 0899-1561/98/0001$0005-0013 / \$ 4.00+\$ .50$ per page. Paper No. 13508 .
}

procedure (Katz and $\mathrm{Li}$ 1996). Interface property characterization in terms of chemical and frictional bond strengths is accomplished by interpreting the pullout test data via the model developed by Leung and $\mathrm{Li}$ (1991), adopted here for simplicity and accuracy.

The second major issue - fiber rupture-dominates the behavior of composites with hydrophilic fibers, and is usually attributed to the small diameter and high bond strength of these fibers. Fiber rupture has been related to fiber strength, embedment length, frictional bond strength, and fiber inclining angle to crack plane (Maalej et al. 1995). The resulting crack bridging law therefore takes explicit account of fiber rupture, assuming that fiber strength in composites is the same as that determined in standard strength tests in which fibers are straightly aligned and gripped with a rigid bond (e.g., ASTM D 2101-94).

This assumption, equating fiber strength from a standard test to that under an in-situ crack bridging condition, may result in severe inaccuracy in the model prediction of composite properties. The difference in boundary conditions can result in lower apparent in-situ fiber strength in composites than in the standard tests due to flexural tensile failure or flexural compressive failure of fibers associated with the bending moment when the fiber axis is inclined at an angle to the crack plane. This type of moment was shown to cause fiber strength reduction for glass fiber obliquely embedded in polyester matrix (Piggott 1974). Furthermore, an Aramid fiber has a one-tenth lower strength in compression than it does in tension (Kozey et al. 1995). Therefore, such fibers may fail by compressive crushing under a bending moment even when subjected to low tensile loads. Hence, for high-strength hydrophilic fiber composites, it is critical to clarify any apparent strength reduction of fibers to properly determine the bridging stress on crack planes.

The ultimate goal of this research is to establish a design methodology for composites using high-strength hydrophilic fibers. Toward this goal, the present study addresses the characterization of both fiber-matrix interfacial properties and fiber rupture employing single-fiber tests with a PVA fiber. The results of these tests allow the quantitative evaluation of specific micromechanical parameters: interfacial bond strengths and apparent fiber-strength reduction factor.

\section{INTERPRETATION METHODS OF FIBER-MATRIX INTERFACE PROPERTY}

\section{Interface Property of Hydrophilic Fiber}

Fibers with a hydrophilic nature generally show high bond strength with cement matrix. For example, the high bond 
strength of PVA fibers was deduced by the microscopic observation of composite fracture surfaces in which a much shorter protruded fiber length was found for PVA fibers than for glass and polypropylene fibers ("PVA" 1986). This high bond strength is attributed to hydroxyl groups on the carbon backbone and resulting strong hydrophilic characteristics of PVA fibers (Akers et al. 1989). The hydroxyl groups lead to a strong hydrogen intermolecular bond (Akers et al. 1989), and therefore contribute to strong chemical bonding between PVA fibers and cement hydration products.

Some quantitative evidence of high bond strength has been experimentally reported for hydrophilic fibers in previous works, while their interface performances have not been precisely evaluated. For example, an apparent bond strength of 4.5 MPa was reported for an Aramid fiber (trade name Kevlar $49)$, whereas bond strengths of 0.16 and $1.02 \mathrm{MPa}$ were determined for a nylon and Polyethylene fiber, respectively, in the same study ( $\mathrm{Li}$ et al. 1990). These data suggest that fibers with hydrophilic surface characteristics have a bond strength several times to an order of magnitude higher than that for fibers with hydrophobic characteristics. No distinction was made between a chemical and frictional bond.

\section{Micromechanical Model for Interpretation of Interface Properties}

Fiber-matrix interface properties are experimentally determined through a load-displacement relation of a fiber pulled out of a matrix. A typical load-displacement curve is shown in Fig. 1. This profile is comprehensive for fiber/matrix systems having superior chemical bonding to friction bonding when no fiber rupture is involved. The profile in Fig. 1 consists of two parts: (1) A debonding process; and (2) a pulling-out process. In the debonding process, the chemical bond is broken and replaced by a frictional bond along the embedment length. The debonding process is followed by the pulling-out process (at $P_{b}$ in Fig. 1), which occurs just after a peak load $P_{a}$ is reached and the embedment length is fully debonded. The load drop from $P_{a}$ to $P_{b}$ signifies unstable propagation of the debonded zone along the interface. This mechanism was investigated in detail by Leung and $\mathrm{Li}$ (1991).

Since debonding governs the ascending portion of the loaddisplacement curve, the conditions for debonding should be theoretically clarified. For this purpose, numerous models have been proposed. These models can be classified into two major categories, depending on the basic assumption of the condition for debonding, namely, a strength-controlled model (Goplaratnam and Shah 1985; Naaman et al. 1991; Leung and $\mathrm{Li}$ 1991) and a fracture controlled model (Stang and Shah 1986;

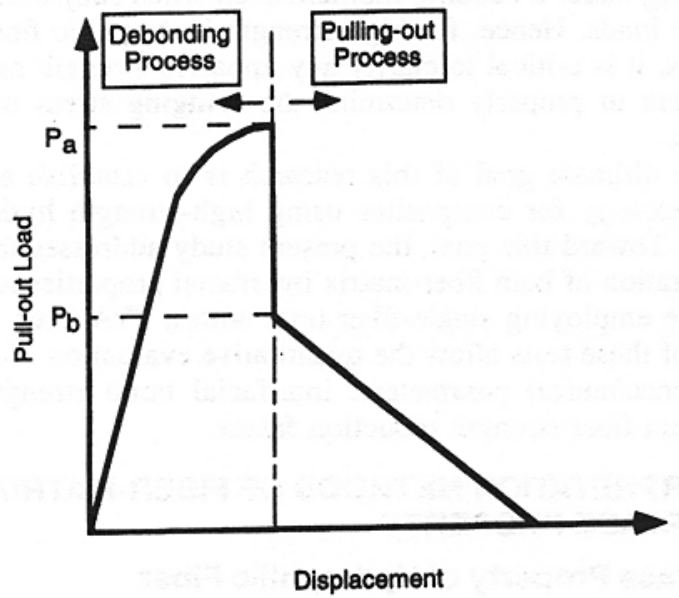

FIG. 1. Schematic of Load-Displacement Relation for Fiber Pulled out of Matrix
Morrison et al. 1988; Gao et al. 1988; Stang et al. 1990; Leung 1992). Assumed shear-stress distribution near the debonding tip is shown in Fig. 2. In the strength-controlled model [Fig. 2(a)], the debonding zone is assumed to propagate when shear stress overcomes a critical value $\tau_{s}$ at a debonding tip; hence, interface performance is represented by frictional bond strength $\tau_{i}$ and chemical bond strength $\tau_{s}$. In the fracture-controlled model [Fig. 2(b)], the debonding zone extends if the energy release rate reaches a critical value $\Gamma$; thus, the specific interfacial parameters are $\tau_{i}$ and $\Gamma$.

The strength-based model proposed by Leung and $\mathrm{Li}$ (1991) is adopted in this study. In their model, $\tau_{s}$ and $\tau_{i}$ can be determined by using the following expressions with values of $P_{a}$ and $P_{b}$ (Fig. 1) experimentally measured from single-fiber pullout tests.

$$
\begin{gathered}
\tau_{i}=\frac{P_{b}}{\pi d_{f} L_{f}} \\
{\left[\left(\frac{\tau_{s}}{\tau_{i}}\right)^{2}-\left(\frac{\tau_{s}}{\tau_{i}}\right)\right]^{1 / 2}-\cosh ^{-1}\left(\frac{\tau_{s}}{\tau_{i}}\right)^{1 / 2}=\left(\frac{P_{a}}{P_{b}}-1\right) \frac{2 \rho L_{f}}{d_{f}}}
\end{gathered}
$$

where

$$
\rho^{2}=\frac{2 G_{c} E_{c}}{\left[V_{m} E_{m} E_{f} \log \left(\frac{2 R^{*}}{d_{f}}\right)\right]}
$$

Undefined variables in formulae are listed in Appendix IV. The criteria for this theory selection was (1) to be as simple as possible; (2) to evaluate single-fiber pullout test results maintaining physical meaning; and (3) to reproduce the loaddisplacement curve of the pullout fiber. The last two criteria are obvious. The first criterion is necessitated by the ultimate research goal to establish a design methodology for hydrophilic fiber composites. This composite design goal requires many additional steps beyond the current stage, which focuses on a single fiber pulled out normal to a matrix crack plane. The model represented by (1) and (2) appears to satisfy these

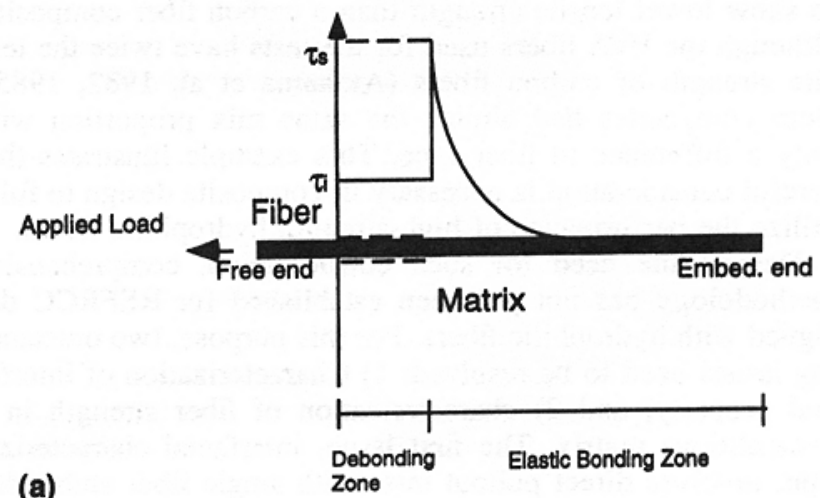

(a)

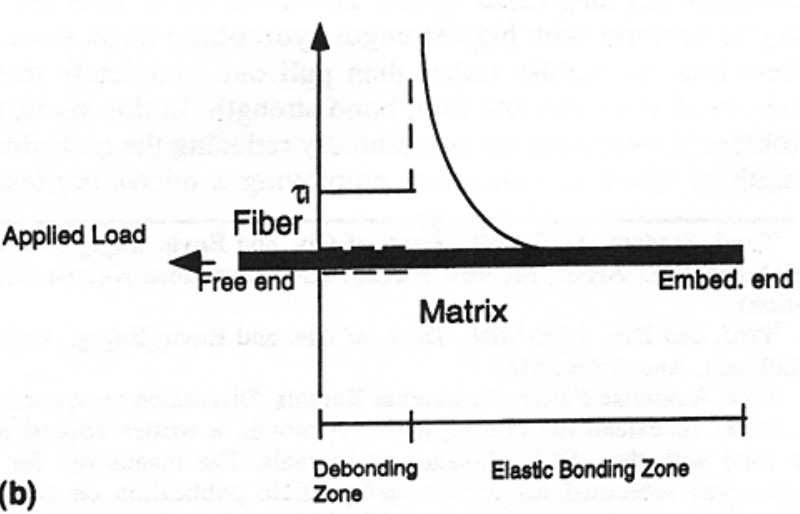

(b)

FIG. 2. Shear Stress Distribution along Embedded Fiber: (a) Strength Control; (b) Fracture Control Distribution 
criteria. The validation of this model is discussed in more detail in a subsequent section.

\section{APPARENT STRENGTH OF POLYMERIC FIBER IN CEMENTITIOUS COMPOSITES}

Fig. 3 shows a fiber with its axis aligned obliquely (at angle $\phi)$ to the normal of the crack plane. For a crack-bridging fiber, the bridging load $P$ is resisted by interfacial shear stresses as well as local interacting forces between the fiber and matrix at the exit point. Such local forces can be described by matrix reaction force $R$ and friction force $\mu R$, or by a snubbing coefficient $f$ when the fiber is flexible and the interaction can be represented by a rope passing over a Euler's friction pulley (Li et al. 1990). This coefficient has the effect of increasing the fiber bridging force $P$ at any given crack opening, whether the fiber eventually fails by rupture or pulls out of the matrix.

When the embedment length is long enough, or if the interfacial bond properties are high enough, fiber rupture may result when fiber strength is exceeded. For an inclined fiber, additional stress is induced by bending. A number of micromechanical fiber/matrix interactions may occur at the position where the fiber exits the matrix. Some likely scenarios will be discussed later together with experimental observations. Whatever the micromechanisms are, experiments of inclined fiber (pull-to-rupture) strength test in cementitious matrix show a clear drop of apparent strength as a function of inclined angle $\phi$ (test data are subsequently shown). For simplicity of data interpretation and for later implementation into a composite model (with randomly oriented fibers), the following expression is used for the apparent fiber strength:

$$
\sigma_{f u}=\sigma_{\rho_{\mu}}^{n} e^{-f^{\prime} \phi}
$$

where $f^{\prime}=$ apparent fiber strength reduction factor, in which $f^{\prime}>0$ is determined by fitting to experimental data of $\sigma_{\mu u} / \sigma_{f u}^{n}$ plotted against $\phi$. The nominal fiber strength $\sigma_{\mu}^{n}$ is defined as the rupture strength of the fiber pulled-to-rupture at $\phi=0^{\circ}$ in a cementitious matrix, and as will be subsequently shown with test data, is lower than the fiber strength determined from a standard test with fiber end fixed (no debonding from matrix).

\section{EXPERIMENTAL PROGRAM}

\section{Single-Fiber Pullout Bond Test}

To examine the properties of interface between matrix and hydrophilic fibers, single-fiber pullout bond tests are conducted in this study. A specimen of these pullout bond tests is

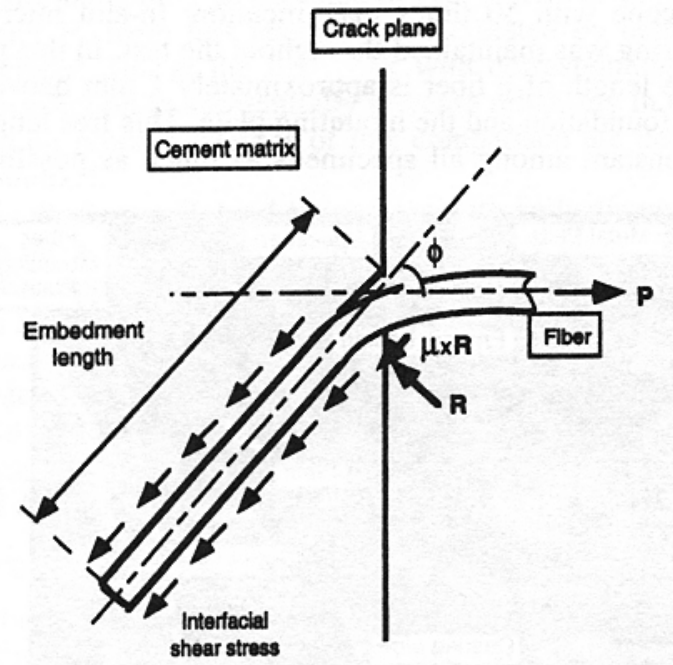

FIG. 3. Bridging of Obliquely Aligned Fiber across Crack Plane illustrated in Fig. 4. For these tests, embedment length $l_{e}$ should be restricted to less than the critical embedment length $l_{\text {crit, }}$ beyond which an embedded fiber ruptures as the pullout load increases rather than slides out of the matrix. $l_{\text {crit }}$ is given by (Kanda and $\mathrm{Li}$, under review, 1997):

$$
l_{\text {crtit }}(\phi)=L_{c i}\left[e^{-\left(s+f^{\prime}\right) \phi}-\xi\right]
$$

where

$$
L_{c l}=\frac{d_{f} \sigma_{f u}^{n}}{4 \tau_{i}}, \xi=\frac{\sigma_{d s}}{\sigma_{\rho u}^{n}}, \sigma_{d s}=2(1+\eta)\left(\frac{\tau_{s}}{\rho}\right), \eta=\frac{V_{f} E_{f}}{\left(1-V_{f}\right) E_{m}}
$$

It can be seen in (4) that the snubbing effect (represented by $f$ ) and the apparent strength effect (represented by $f^{\prime}$ ) for inclined fibers combine to reduce the critical embedment length $l_{\text {crit. }}$. For these bond tests, inclining angle $\phi$ is set to zero, and the specimens are fabricated according to the technique for microfibers described in $\mathrm{Katz}$ and $\mathrm{Li}$ (1996) to ensure accurate fiber alignment. The specimens are set up for loading as in Fig. 5, where specimens are mounted with superglue onto aluminum plates. In this mounting process, a small amount of glue is carefully pasted on the four corners of specimens such that the superglue never affects the fiber pullout behavior. This mounting condition and horizontal specimen size minimizes any artificial confining effect that can be estimated via the magnitude of $R^{*}$ in (2), and is considered minor in theory. Tensile loads of specimens are measured with miniature load cell (1 N capacity), and displacement is measured through actuator head movement. Details of the tests are described in Chan and $\mathrm{Li}$ (1997a).

The pullout bond tests employ two major experimental parameters, fiber type and matrix type. The two types of fibers employed in this test are both PVA and therefore have the same chemical structure. However, they are different in fiber diameter and surface finish. Information of fiber properties is

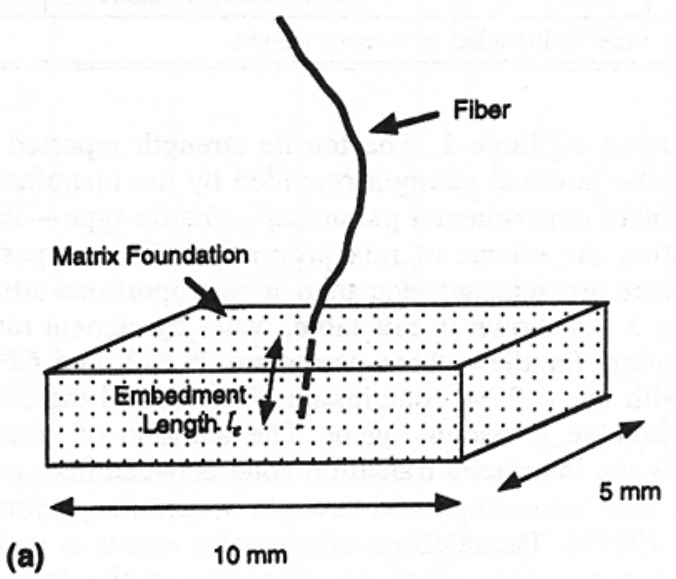

(b)

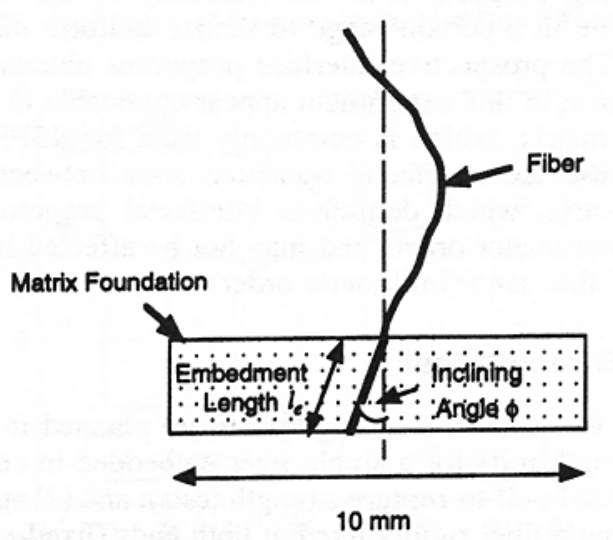

FIG. 4. Single-Fiber Pullout Specimen: (a) Perspective of Specimen; (b) Section of Specimen 


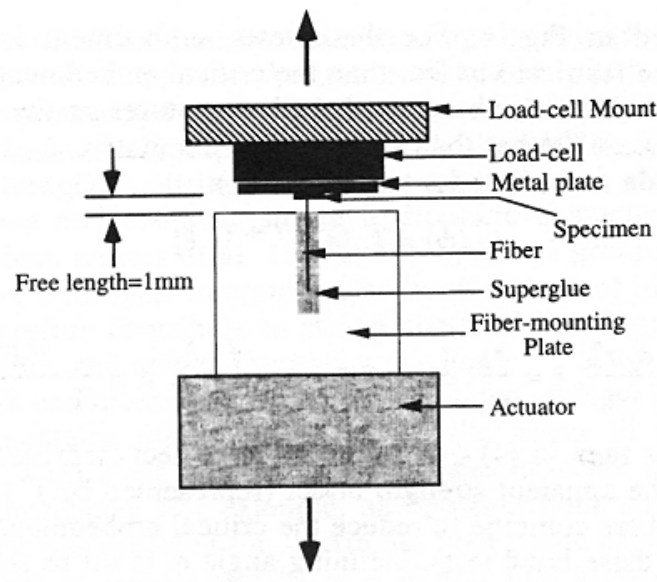

FIG. 5. Single-Fiber Pullout Test Setup

TABLE 1. Fiber Properties

\begin{tabular}{c|c|c|c|c}
\hline \hline & Diameter \\
$(\mu \mathrm{m})$ & $\begin{array}{c}\text { Density } \\
(\mathrm{kg} / \mathrm{L})\end{array}$ & $\begin{array}{c}\text { Tensile } \\
\text { strength } \\
(\mathrm{MPa})\end{array}$ & $\begin{array}{c}\text { Elastic } \\
\text { modulus } \\
(\mathrm{GPa})\end{array}$ & $\begin{array}{c}\text { Tensile } \\
\text { strain } \\
\text { capacity } \\
(\%)\end{array}$ \\
\hline 14 & $(2)$ & $(3)$ & $(4)$ & $(5)$ \\
40 & 1.3 & 2,254 & 60 & 5 \\
\hline
\end{tabular}

TABLE 2. Mix Proportions (Weight Ratios)

\begin{tabular}{c|c|c|c|c|c}
\hline \hline $\begin{array}{c}\text { W/C } \\
(\%)\end{array}$ & $\begin{array}{c}\text { Cement } \\
(2)\end{array}$ & $\begin{array}{c}\text { Silica } \\
\text { fume } \\
(3)\end{array}$ & $\begin{array}{c}\text { Water } \\
(4)\end{array}$ & $\begin{array}{c}\text { Super } \\
\text { plasticizer } \\
(5)\end{array}$ & $\begin{array}{c}\text { Viscous } \\
\text { agent } \\
(6)\end{array}$ \\
\hline $27^{\mathrm{a}}$ & 0.8 & 0.2 & 0.27 & 0.040 & - \\
42 & 1.0 & - & 0.42 & 0.007 & 0.0015 \\
62 & 1.0 & - & 0.62 & 0.007 & 0.0050 \\
\hline
\end{tabular}

"Silica fume is included in cement weight.

summarized in Table 1. The tensile strength reported in this table is the nominal strength provided by the manufacturer.

The other experimental parameter-matrix type-is for investigating the effects of mix proportion. Three types of cement paste are selected, and their mix proportions are shown in Table 2. As shown in this table, water by cement ratios $(w /$ $c$, hereafter) for these three pastes are 27,42 , and $62 \%$. The paste with $w / c=27 \%$ contains microsilica, and the other two pastes involve a viscous agent. The addition of silica fume densifies the interfacial transition zone between fiber and bulk matrix, thus increasing bond strength in certain systems (Chan and $\mathrm{Li} 1997 \mathrm{~b}$ ). The addition of viscosity agents is mainly for processing purposes since the viscosity of the fresh pastes should be in a certain range to realize uniform dispersion of fibers. The prospective interface properties obtained with cement paste in this experiment appear applicable to the case of mortar matrix, which is commonly used for RSFRCCs. This is because the interfacial transition zone between fiber and bulk matrix, which dominates interfacial properties, is very thin (micrometer order) and may not be affected by the presence of fine sand (millimeter order).

\section{Fiber-Strength Test}

Two series of fiber-strength tests are planned in this study: (1) strength tests for a single fiber embedded in cement paste (embedded pull-to-rupture strength tests); and (2) strength tests for a single fiber rigidly fixed at both ends (fixed-end strength tests). The first test series is conducted to investigate apparent fiber strength in cementitious composites. The same single-

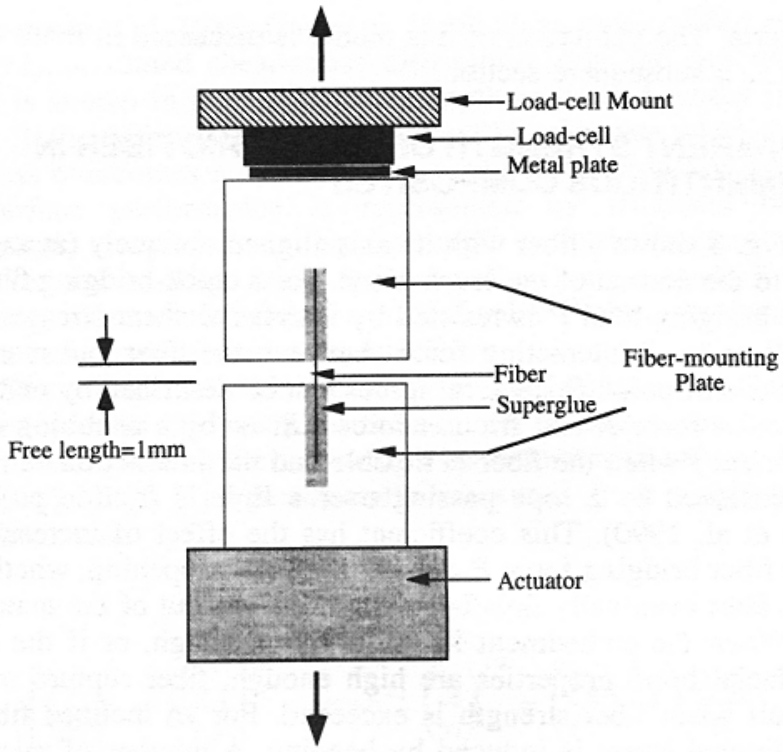

FIG. 6. Single-Fiber Fixed-End Strength Test Setup

fiber pullout specimens and test setup described earlier (Figs. 4 and 5) are adopted. However, these strength tests employ embedment length $l_{e}$ deliberately extended beyond critical embedment length $l_{\text {crit }}$, as determined by (4). These tests are performed with six different fiber-inclining angles $\phi: 0,15,30$, 45,60 , and $75^{\circ}$, using the fibers with a diameter of $14 \mu \mathrm{m}$ and a matrix with $w / c=27 \%$ (details in Tables 1 and 2).

The second strength test series is for examining the effect of boundary conditions on apparent fiber strength (this test result is also used to facilitate interpretation of the bond test results as described in Appendix I). Specifically, a single fiber is aligned between two aluminum plates and mounted to the plates with superglue as shown in Fig. 6, similar to the lower grip of the pullout specimen setup in Fig. 5. Superglue maintains a very strong elastic bond, and prevents the fiber from debonding in the glue. Therefore, combined with the first tests series, these strength tests can reveal the effects of fiber sliding from the matrix on the apparent fiber strength.

\section{RESULTS AND DISCUSSION FOR INTERFACE PROPERTIES}

\section{Test Results}

The actual test setup is illustrated in Fig. 7 for the singlefiber pullout bond test. This picture was obtained by optical microscope with 50 times magnification. In-situ microscopy monitoring was maintained throughout the test. In this picture, the free length of a fiber is approximately $1 \mathrm{~mm}$ between the matrix foundation and the mounting plate. This free length was kept constant among all specimens as much as possible, and

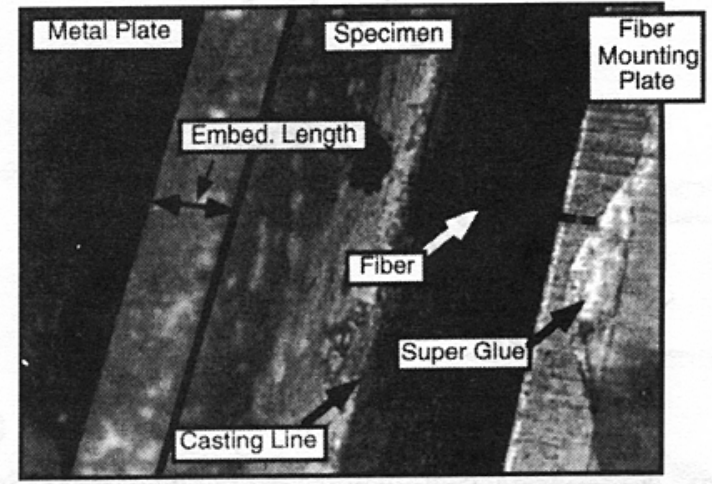

FIG. 7. Actual Setup of Single-Fiber Pullout Bond Test 


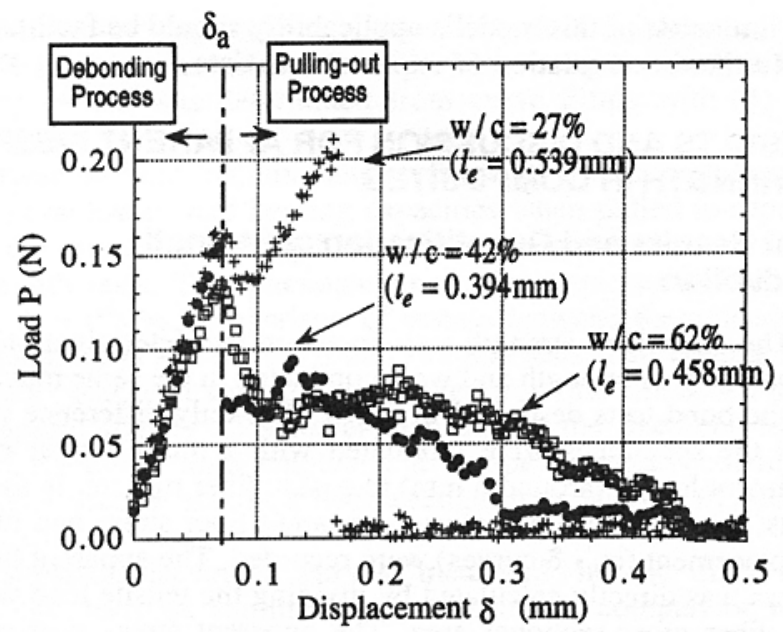

FIG. 8. Test Results of Load-Displacement Relation in Pullout Bond Test

was blackened with ink before loading to distinguish it from the embedded portion. Embedment length $l_{e}$ of a fiber is about $0.5 \mathrm{~mm}$ in Fig. 7. This embedment length varied in the 0.3$1.2 \mathrm{~mm}$ range among specimens due to the difficulty of slicing specimens very thin; the influence of this length will be discussed in the next subsection.

Load-displacement curves for the 14- $\mu \mathrm{m}$-diameter fibers from the pullout bond tests are shown in Fig. 8. This figure mainly illustrates the influence of matrix (the three tests involve fibers with similar but not identical embedment length). In all cases, loads increase almost linearly at the beginning followed by sudden drop after the peaks are reached, as expected in Fig. 1. These drops occur at almost the same displacement independent of matrix $w / c$. However, loads increase again as displacement further increases, contrary to the illustration in Fig. 1. This load increase prior to the final descent leads to the second peak, which can be even higher than the first peak as exemplified by the data for the lower $w / c$ matrix shown in Fig. 8. A similar load increase was observed in other fiber-matrix systems such as in tests with plasma-treated polyethylene fiber ( $\mathrm{Li}$ et al. 1996), and was attributed to a sliphardening process associated with fiber surface abrasion.

According to Fig. 1, the load-displacement relation is characterized by two parameters, peak load $P_{a}$ and load after a sudden drop $P_{b}$, of which results are summarized in Fig. 9. The accuracy of determining these two loads is of concern since the stress drop from $P_{a}$ to $P_{b}$ can be difficult to identify due to slip-hardening as depicted in Fig. 8. The determination of $P_{a}$ and $P_{b}$ from test results was facilitated by comparing displacement data at the first peak with corresponding theoretical prediction to substantially increase reliability in this determination process. Details of this comparison are described in Appendix $\mathrm{I}$.

In Fig. 9, $P_{a}$ and $P_{b}$ tend to increase with embedment length $l_{e}$ while the scatter of the test results is rather large. The data trend was approximated by linear fitting, shown as straight lines in Fig. 9. From the comparison of these straight-line approximations, the following features are observed: (1) Both $P_{a}$ and $P_{b}$ tend to be higher in matrices with $w / c=27$ and $42 \%$ than that with $w / c=62 \%$; (2) $P_{a}$ and $P_{b}$ increase with embedment length $l_{e}$ more rapidly in lower $w / c$ matrices; and (3) the ratio of $P_{a} / P_{b}$ is smaller in lower $w / c$ matrices.

\section{Interpretation of Test Results}

Interfacial bond strength $\tau_{s}$ and $\tau_{i}$ were estimated by using the test results of $P_{a}$ and $P_{b}$ via (1) and (2). First, test results for each specimen were used for calculation, yielding evaluation results of $\tau_{s}$ and $\tau_{i}$ for individual specimens. These cal- culated results were than averaged among specimens to give mean values of $\tau_{s}$ and $\tau_{i}$ of each data set. In this evaluation process, calculation with (1) and (2) employed the different magnitudes of elastic modulus $E_{c}$ for each matrix $w / c$ according to Hirsch (1962). Furthermore, the matrix volume fraction $V_{m}$ was taken as 1 , and the effective cylinder radius $R^{*}$ was assumed to be $2.5 \mathrm{~mm}$, half of the actual specimen width.

As a result of this evaluation, the bond strengths were determined as shown in Fig. 10. This figure illustrates surprisingly high chemical and frictional bond strength. The frictional bond varies from 2.2 to $4.4 \mathrm{MPa}$, whereas the chemical bond ranges from 29 to $37 \mathrm{MPa}$. Such high bond strengths of PVA fibers are quantitatively evaluated through experiments in this study for the first time. The results in Fig. 10 also show that $\tau_{s}$ is rather stable, independent of the $w / c$ of matrix and the two types of PVA fibers, whereas $\tau_{i}$ appears to vary depending on these two parameters. This tendency may be interpreted as follows. Hydrophilic fibers, such as PVA fibers, are expected to show a strong chemical bond with cement matrix, of which strength is principally determined by the surface chemistry of fibers and cement chemistry. These chemical structures were not altered by water content so that $\tau_{s}$ is independent of matrix $w / c$. On the other hand, $\tau_{i}$ is considered to obey a Coulombtype friction law, which is described by a fiber-matrix friction coefficient and residual normal shrinkage stress of matrix onto the fiber surface ( $\mathrm{Li}$ and Stang 1997). Therefore, $\tau_{i}$ may be expected to decrease with the reduction of these two factors. In the tests, lower $\tau_{i}$ was observed as $w / c$ was increased, and may be related to the lower elastic modulus of the high w/c matrix that tends to reduce the normal stress. Furthermore,

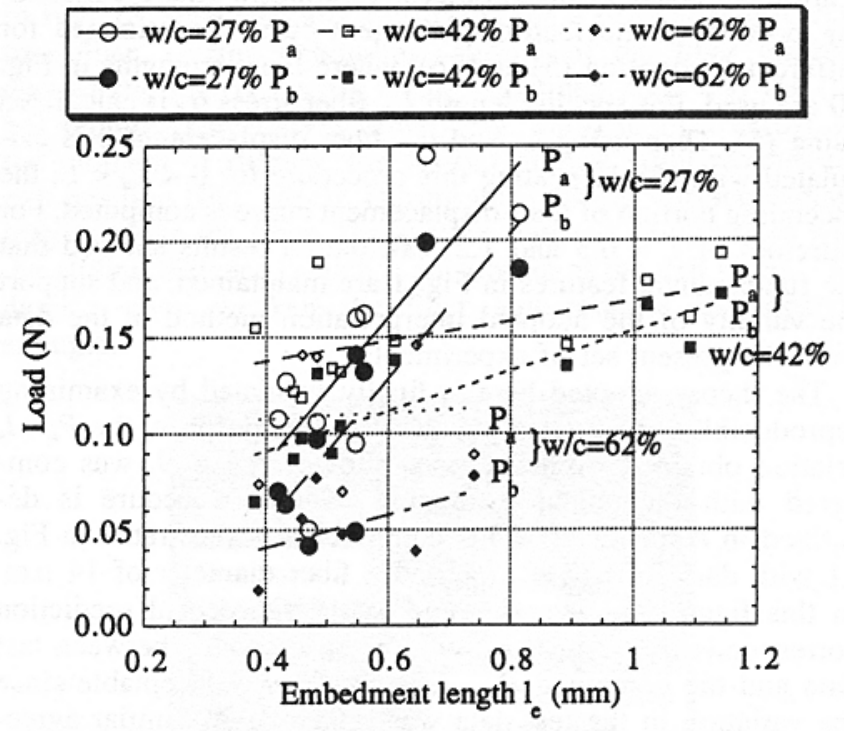

FIG. 9. Summary of Results in Pullout Bond Tests

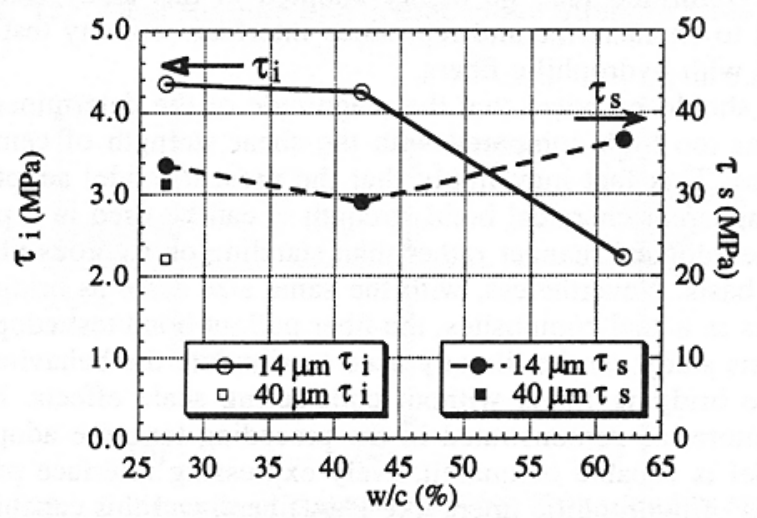

FIG. 10. Estimated Results for Bond Strength 


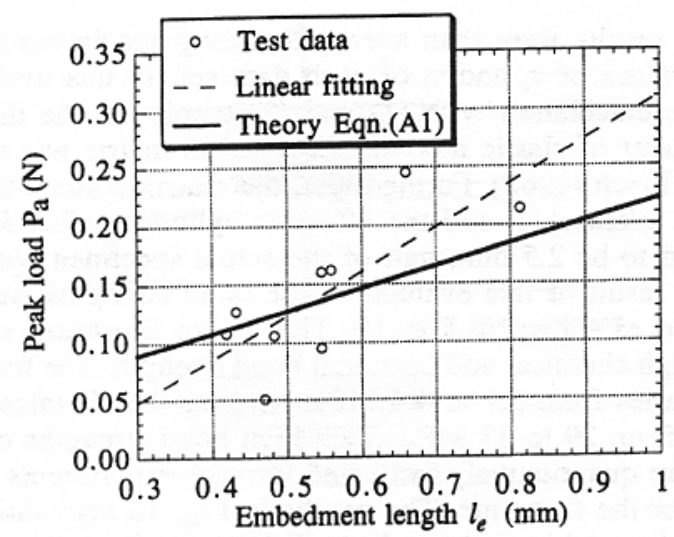

FIG. 11. Comparison of Peak Pullout Load between Test and Theory

increasing porosity of the materials in the interfacial zone may lead to a reduction of the fiber-matrix contact surface, resulting in a lower $\tau_{i}$. The lower $\tau_{i}$ for the $40-\mu \mathrm{m}$ fibers may be a reflection of the one-third lower elastic modulus of these fibers than that of the $14-\mu \mathrm{m}$ fibers (see Table 1).

In the bond determination tests, fiber embedment length varied between 0.3 and $1.2 \mathrm{~mm}$, as described in the previous subsection. It should be verified that this variation should not lead to contamination of the interpreted bond properties. In principle, the difference of embedment length, $l_{e}$, is accounted for in (1) and (2), thus not being a concern, provided that the fundamental features in the load-displacement relation in Fig. 1 are maintained within $l_{e}$ ranges. To confirm this, the ascending portion of the load-displacement curve is estimated for different $l_{e}$ adopting (5) and (6), where bond strengths in Fig. 10 are used. For specific length $L_{d}$, fiber stress $\sigma_{d}$ is calculated using (5). Then using $L_{d}$ and $\sigma_{d}$, fiber displacement $\delta$ is calculated with (6). Repeating this procedure for $0<L_{d}<l_{e}$, the ascending portion of load-displacement curve is computed. For extremes of $l_{e}=0.3$ and 1.2 , calculation results showed that the fundamental features in Fig. 1 are maintained, and support the validity of the adopted interpretation method of the data from the present set of experiments.

The theory adopted here is finally validated by examining reproducibility of the test results. For this purpose, the $P_{a}-l_{e}$ relation obtained from the tests, shown in Fig. 9, was compared with theoretical estimation, whose procedure is described in Appendix II. This comparison is illustrated in Fig. 11 with data for $w / c=27 \%$ and a fiber diameter of $14 \mu \mathrm{m}$. In this figure, the overall trend in the theoretical prediction corresponds to the test results. The discrepancy between test data and the computed $P_{a}-l_{e}$ is considered acceptable since the variation in the test data was rather large. Similar agreement was also observed in other data sets. Consequently, it was confirmed that the theory adopted in this study can be used to characterize and reproduce interface property test results with hydrophilic fibers.

It should be noted that the magnitude of the determined $\tau_{s}$ seems too high compared with the shear strength of cement pastes. This fact may imply that the present model adopting the apparent chemical bond strength $\tau_{s}$ can be used in a phenomenological manner rather than standing on rigorous physical basis. Nevertheless, with the same size scale as bridging fibers in actual composites, the fiber pullout bond test adopted in this study can be directly used to simulate the behavior of those bridging fibers without considering scale effects. Furthermore, as demonstrated in the preceding text, the adopted model is capable of quantitatively expressing interface properties of hydrophilic fibers like PVA fibers, and this capability is sufficient for the purpose of this study. However, clarifying the limitation of this model's applicability should be facilitated by further accumulation of experimental data.

\section{RESULTS AND DISCUSSION FOR APPARENT FIBER STRENGTH IN COMPOSITES}

\section{Test Results and Quantification of Strength Reduction}

The embedded strength tests focus on the determination of apparent fiber strength and were conducted in the same manner as the bond tests described in Fig. 7. The only difference was that the specimens were fabricated with a much longer embedment length (around $5 \mathrm{~mm}$ ) to ensure fiber rupture. In these tests, the relationships between apparent fiber stress and fiber displacement ( $\sigma_{a}-\delta$ curves) were recorded. The apparent fiber stress was directly calculated by dividing the tensile load with the fiber cross-sectional area. The apparent stress primarily represents fiber stress in the free portion and its peak was identified as the apparent fiber strength $\sigma_{f u}$ in the tests.

Typical results in the embedded strength tests are illustrated in Fig. 12, where the $\sigma_{a}-\delta$ relation is depicted up to fiber rupture for different inclining angles $\phi$. Fibers tend to rupture at lower stress as $\phi$ increases. For highly inclined fibers $(\phi>$ $60^{\circ}$ ), multiple load drops were observed. This load drop is attributed to matrix spalling as evidenced by microscopy observations (Fig. 13). Fig. 13 is a picture of a fiber showing a ruptured end after the strength test at $\phi=75^{\circ}$. The portion originally embedded in matrix is distinguished from the free portion by its brighter color since the free portion was blackened before the tests. Fig. 13 reveals that the fiber ruptured at the originally embedded point $100 \mu \mathrm{m}$ into the matrix. Furthermore, Fig. 14 illustrates a spall hole revealed by the movement of the fiber exit point.

The descending trend of apparent fiber strength $\sigma_{f u}$ was clearly found in test results with an increase of inclining angle $\phi$. These results are summarized in Fig. 15, which reveals that

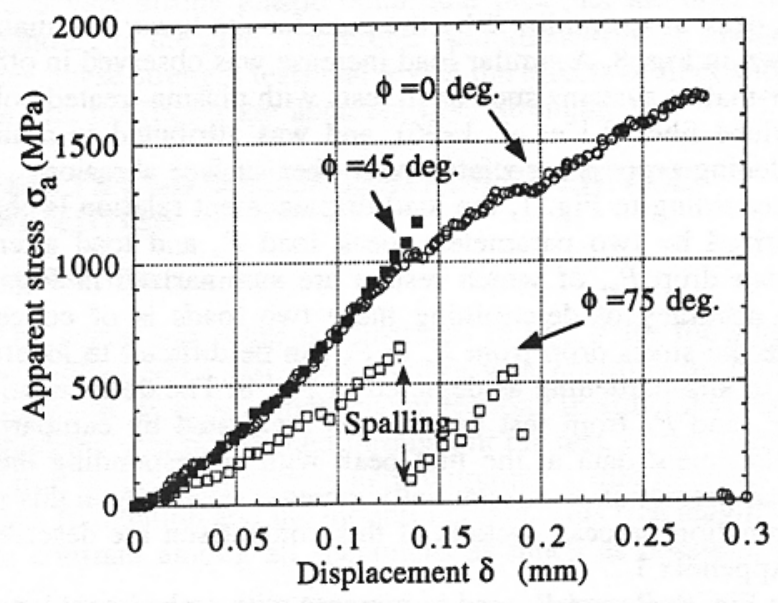

FIG. 12. Results of Embedded Strength Tests

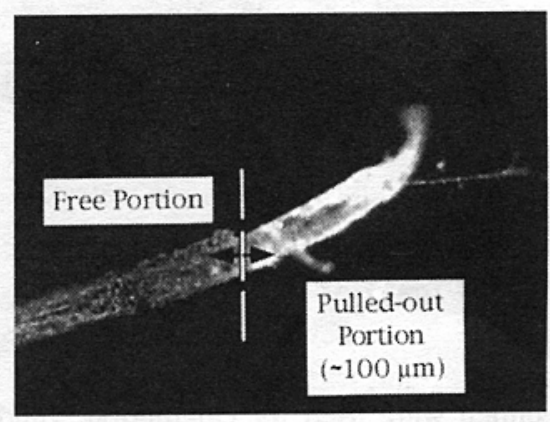

FIG. 13. Rupture End of Pulled-Out Fiber with Spalling 
the inclining angle effect is serious so that $\sigma_{f u}$ decreases by $40 \%$ as $\phi$ increases from 0 to $75^{\circ}$. The strength-reduction factor $f^{\prime}=0.3$ was determined from curve fitting with (3) ( $\phi$ : radian).

Even without inclining angle effects, hydrophilic fibers tend to show lower load bearing capacities when pulled to rupture in the embedded strength test than under the standard fiber strength tests. This phenomenon is also depicted in Fig. 15, for $\phi=0^{\circ}$, by comparison of results between the embedded strength tests and the fixed-end strength tests. This comparison reveals that fiber strength determined from the embedded tests is approximately $15 \%$ lower than that in the fixed-end tests. The fixed-end tests employed a similar boundary condition widely used in standard fiber strength tests such as in ASTM D 2101.

Stress-displacement curves measured in the fixed-end strength tests are summarized in Fig. 16. By comparison with Fig. 12, these curves show twice the stiffness of those from the embedded strength tests. This is a direct result of the difference in fiber grip-end conditions between these two test series, i.e., it is due to debonding of the fiber-gripped-end in

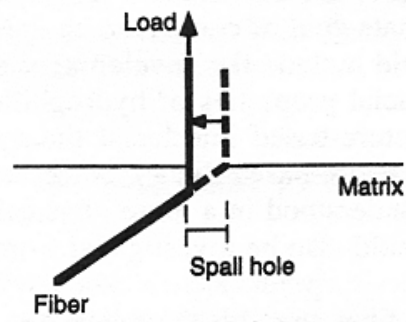

FIG. 14. Spalling of Matrix Foundation

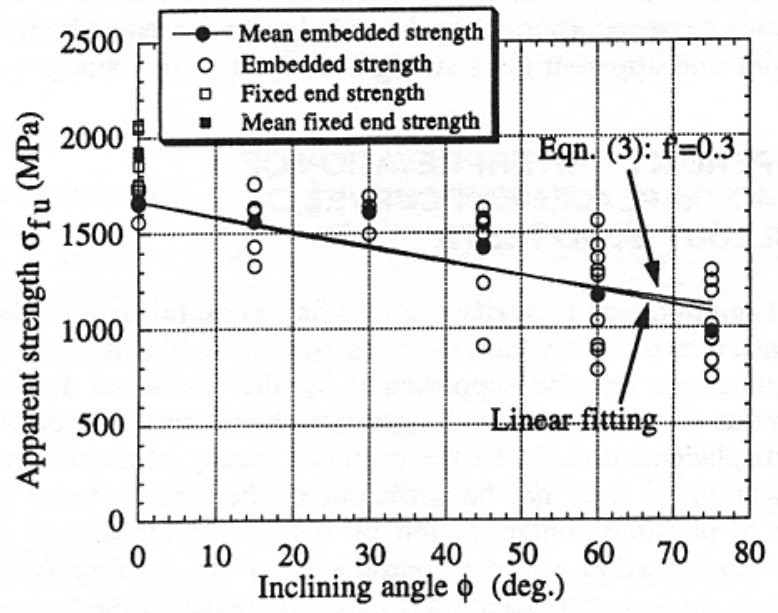

FIG. 15. Inclining Effect on Apparent Fiber Strength

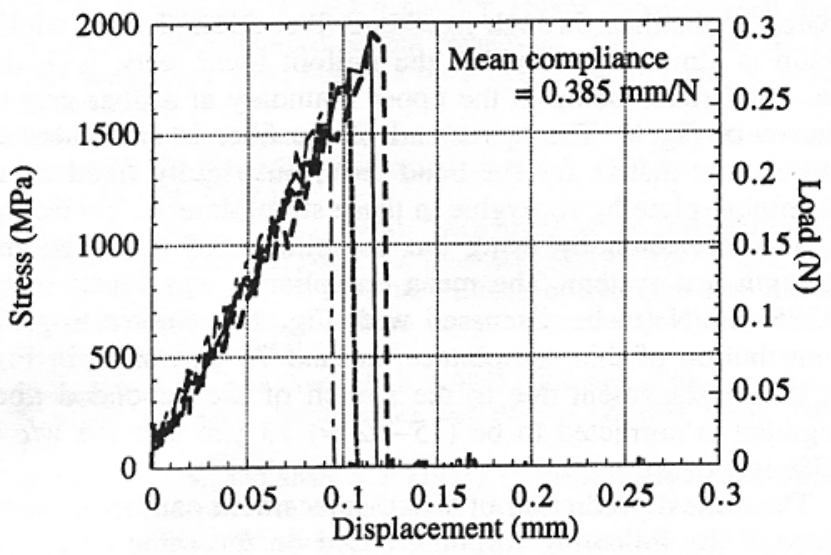

FIG. 16. Fixed-End Strength Test Result the matrix in the embedded test. This difference is likely to affect fiber strength test results as expected earlier. The plausible mechanism of this influence is discussed in the next subsection.

\section{Possible Mechanisms of Fiber-Strength Degradation}

There are several possible micromechanisms that individually or in combination act to lower the apparent strength of PVA fibers as observed in Fig. 15 and discussed in the last subsection. In this section the writers discuss these mechanisms, including abrasion damage, spall damage, and flexure, as well as plausible supporting evidence for each mechanism.

In the embedded strength test, it was observed that most fibers ruptured at a point originally embedded inside the matrix. This can be seen in the optical micrograph of the ruptured end of the fiber $\left(\phi=45^{\circ}\right)$, shown in Fig. 17. For this fiber, the uninked portion measures approximately $50 \mu \mathrm{m}$. This phenomenon appears to not be dependent on the inclination angle, and is revealed even for nominally $\phi=0^{\circ}$ fibers. Using (5) and (6), and ignoring the local fiber/matrix interacting effects, the debond length $L_{d}$ and fiber displacement $\delta$ (at exit point) due to stretching of the debonded segment can be determined at the fiber rupture load. For the set of $\phi=45^{\circ}$ fibers, it was found that $L_{d}=0.9 \mathrm{~mm}$ and $\delta=62 \mu \mathrm{m}$ on the average. ( $\delta$ is expected to be even smaller if the snubbing effect is taken into account.) The debond length is clearly much smaller than the full embedment length $(5 \mathrm{~mm})$, so that the fibers must have ruptured during the debonding process. The calculated fiber displacement is slightly larger than the average measured value of the uninked ruptured end of $43 \mu \mathrm{m}$ for this set of fibers. These observations suggest the fiber rupture scenario shown in Fig. 18, schematically illustrated for a straight pull-to-rupture fiber. During debonding, sliding of the fiber material against the matrix occurs in the debonded zone and the (tobe) rupture point moves down [Fig. 18(a) to Fig. 18(b)]. At the moment of rupture, the (to-be) rupture point reaches the exit position [Fig. 18(c)]. During this process, surface damage may occur in the fiber. The surface damage likely scales with the sliding distance, which is maximum at the exit point. This damage may weaken the fiber and reduces its apparent strength.

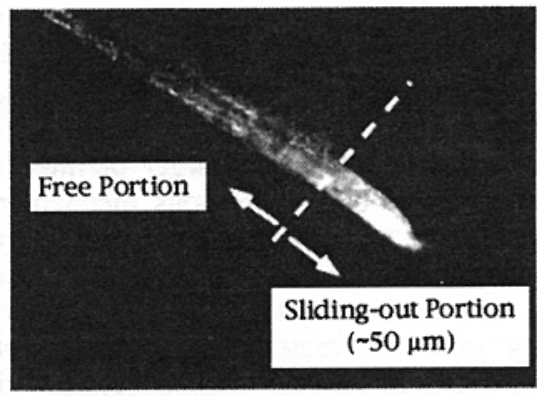

FIG. 17. Fiber Rupture End in Embedded Strength Tests $(\phi=$ $\left.45^{\circ}\right)$

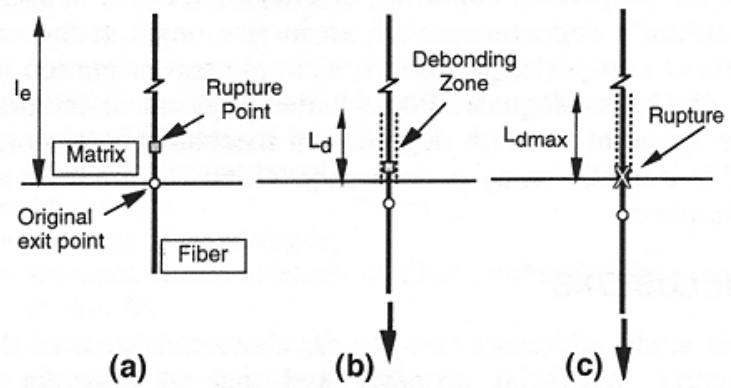

FIG. 18. Schematic of Rupture of Fiber Embedded in Matrix: (a) Load $P=0$; (b) Load $P>0$; (c) Load $P=$ Failure Load 
Surface damage in the form of abrasion in synthetic fibers has been observed by $\mathrm{Li}$ et al. (1988), and has been associated with a slip-hardening effect on fiber pullout curves. Slip-hardening was also observed in the PVA fibers in the current study, as discussed earlier in connection with Fig. 8. This hypothesis of apparent strength degradation due to surface abrasion is further supported by the comparison of apparent fiber strength between the embedded strength and the fixed-end strength tests, both carried out at $\phi=0^{\circ}$. The reduction from 1,900 to $1,700 \mathrm{MPa}$ can only be attributed to this influence of fiber end condition.

Matrix spalling was observed for the high-angle fibers (Fig. 12). Matrix spalling may introduce a sudden load on the fiber when the matrix foundation supporting the fiber is suddenly removed. It is plausible that such sudden loading causes damage to the fiber, such as by local buckling on the underside of the fiber. The test results show that the apparent strength rarely exceeds the load level associated with spalling (see Fig. 12). In addition, spalling releases a segment of embedded fiber and suggests that a longer uninked rupture end could result. Microscopic observations place the length of the uninked rupture end at approximately $110 \mu \mathrm{m}$ (averaged) in specimens showing spall at $\phi=75^{\circ}$.

Finally, even without any weakening of the fiber, simple flexure of inclined fibers must introduce tensile stress on one side of the fiber at the exit point where the curvature is largest. The exact curvature will depend on the inclined angle $\phi$, but also the foundation stiffness (dependent on the cement matrix property), and fiber stiffness and diameter. If simple flexure were responsible for the apparent fiber-strength degradation, the strength should decrease linearly with an inclined angle. Fig. 15 shows that a linear fit to the data is not entirely unreasonable, which lends support to the influence of flexureinduced tensile stress on the apparent fiber strength. From Fig. 15 , comparison between the apparent fiber strength at $\phi=0^{\circ}$ and $75^{\circ}$ indicates a flexure-induced tensile stress of $650 \mathrm{MPa}$. Based on this simple flexure concept, the stress on the compression side of the bent fiber will be approximately (1000$650=) 350 \mathrm{MPa}$ at the fiber rupture load of $1000 \mathrm{MPa}$ (average value for the $\phi=75^{\circ}$ fibers). This stress is still in tension, implying that compression failure of the fiber is ruled out, at least if no other micromechanisms as discussed earlier are simultaneously operating.

A careful observation of the data in Fig. 15, however, seems to suggest a bilinear decay of apparent fiber strength. The strength reduction rate at small inclined angles $\left(\phi<45^{\circ}\right)$ is low, but becomes much faster at larger inclined angles. It is plausible that all three of the previous mechanisms may operate simultaneously, and may in fact interact synergistically rather than simply additive. For example, it is likely that surface damage may increase with flexing of the fiber that bears compressively onto the matrix. This bearing pressure and therefore fiber abrasion damage should increase with inclining angle. And, of course, it is this same bearing pressure that leads to matrix spalling at higher inclined angles.

For the purpose of capturing this negative effect of apparent fiber-strength degradation with an incline angle in theoretical models of composite properties, a simple representation in the form of (3) is adequate. For a better physical understanding of the apparent strength degradation mechanisms, a more detailed systematic study is necessary. This is beyond the scope of this paper.

\section{CONCLUSIONS}

This study addresses two issues: characterization of the fiber-matrix interfacial property and that of apparent fiber strength to serve the ultimate research goal-establishing a micromechanics-based composite design methodology with hydrophilic high-strength fibers. PVA fiber was found to have a surprisingly high apparent chemical bond $\left(\tau_{s} \approx 30-40 \mathrm{MPa}\right)$ and frictional bond $\left(\tau_{i} \approx 2.2-4.4 \mathrm{MPa}\right)$ strength. The chemical bond strength was relatively stable, independent of the waterto-cement ratio of the matrix and fiber type tested, contrary to the behavior of the friction bond strength. The micromechanical theory adopted here was found capable of reasonably interpreting and reproducing the single-fiber pullout test data. Straight pull-to-rupture tests revealed that the apparent strength of (14 $\mu \mathrm{m}$ diameter) PVA fibers in cementitious composites is lower $(\approx 1,700 \mathrm{MPa})$ than that under a fixed-end condition $(\approx 1,900 \mathrm{MPa})$. The apparent strength was further reduced with an inclining angle of embedded fiber to the crack plane, dropping to approximately $1,000 \mathrm{MPa}$ at $75^{\circ}$. This effect was captured by a simple phenomenologica model in this study, which introduces the apparent strength-reduction factor $f^{\prime}$ found to be approximately 0.3 for the fiber/matrix system investigated. The combined effects of high bond strength and degraded fiber strength will likely contribute to a composite performance less than that expected from a high-performance fiber.

The preceding two issues should be further examined to achieve the ultimate goal of composite engineering. These further studies should include the development of methodologies to control interfacial properties of hydrophilic fibers. For this purpose, the fracture-based interfacial theory will be needed instead of the strength-based theory, since the interface properties are to be understood in a more physically rigorous way. Fiber rupture should also be investigated from a more detailed micromechanical viewpoint. Such a study will lead to the enhanced usage of fiber strength in composites.

Finally, it should be clear that any micromechanics-based model for the mechanical behavior of hydrophilic fiber composites must incorporate the knowledge on interface bond behavior and apparent fiber strength derived in this study.

\section{APPENDIX I. INTERPRETATION OF LOAD-DISPLACEMENT CURVES OF PULLOUT BOND TESTS}

It is important to verify that the first peak in load-displacement curves corresponds to $P_{a}$ as shown in Fig. 8. This correspondence may be supported if $\delta_{a}$, the measured displacement at the first peak load, agrees with theoretical prediction of displacement at $P_{a}$. However, the accuracy of displacement measurement may not be sufficient in the current bond tests due to possible contamination by the elastic elongation of a free-fiber portion and the compliance of the loading system. Hence, the raw displacement data are corrected at the first peak load point by eliminating the contribution of these two contamination sources. The displacement contribution to be eliminated is obtained through fixed-end fiber-strength tests whose setup is almost the same as the pullout bond tests, with the only difference being in the upper boundary at a fiber grip as shown in Fig. 6. The upper end of the fiber is embedded in the cement matrix for the bond tests, but rigidly fixed to an aluminum plate by superglue in these strength tests. Therefore, $\delta_{a}$ was corrected by using the compliance of the fixed-end strength test system. The mean compliance was found to be $0.385 \mathrm{~mm} / \mathrm{N}$ (to be discussed with Fig. 16). Subtracting the contribution of this compliance, around $75 \mu \mathrm{m}$ of $\delta_{a}$ in Fig. 8 , the displacement due to the stretch of the debonded fiber segment is corrected to be $(75-62=) 13 \mu \mathrm{m}$ (for the $w / c=$ $27 \%$ test data).

Theoretical prediction of this displacement can be obtained through the following formula, based on the same analytical assumption as (1) and (2) (Li and Leung 1992): 


$$
\sigma_{d}=\frac{\left[2\left(\frac{2 L_{d}}{d_{f}}\right) \tau_{i} \cosh Z+2\left(\frac{\tau_{s}}{\rho}\right) \sinh Z\right]}{(1-\alpha) \cosh Z+\alpha}
$$

where

$$
\begin{gathered}
Z=2 \rho\left(l_{e}-L_{d}\right) / d_{f}, \alpha=V_{f} E_{f} / E_{c} \\
\frac{\delta}{d_{f}}=\left(\frac{\sigma_{d}}{E_{f}}\right)\left(\frac{2 L_{d}}{d_{f}}\right)-\frac{\tau_{i} E_{c}\left(\frac{2 L_{d}}{d_{f}}\right)^{2}}{E_{f} V_{m} E_{m}}
\end{gathered}
$$

From (5), the debond length $L_{d}$ at peak load $P_{a}$ can be determined since the bond strength $\left(\tau_{i}\right.$ and $\left.\tau_{s}\right)$ and fiber stress $\sigma_{d}$ are now known. Substitution of the resulting $L_{d}$ into (6) allows $\delta$ at $P_{a}, \delta_{a}$, to be calculated. As a result of this estimation, $L_{d}$ and $\delta_{a}$ were found to be 554 and $18 \mu \mathrm{m}$ for the specimen with $w / c=27 \%$ in Fig. 8 .

Consequently, these theoretical predictions, $L_{d}=554 \mu \mathrm{m}$ and $\delta_{a}=18 \mu \mathrm{m}$, were found to closely correspond to the actual embedment length of the specimen $(539 \mu \mathrm{m})$ and the corrected displacement test data $(13 \mu \mathrm{m})$. The test results of other specimens showed similar agreement with theory. Therefore, it can be concluded that the determination of $P_{a}$ and $P_{b}$ was valid in this study.

\section{APPENDIX II. THEORETICAL PREDICTION OF PEAK LOAD}

Theoretical prediction of peak load $P_{a}$ was conducted by employing (5), where averaged results of $\tau_{s}$ and $\tau_{i}$ in Fig. 10 were used. In (5), fiber stress $\delta_{d}$ is expressed as a function of debonding length $L_{d}$ and embedment length $l_{e}$. For fixed $l_{e}$, the maximum stress, which yields an estimated value for $P_{a}$, was obtained in a $\sigma_{d}-L_{d}$ curve using numerical computation.

\section{ACKNOWLEDGMENTS}

Part of this work was supported by the National Science Foundation, through Research Grant CMS-9601262 to the ACE-MRL at the University of Michigan. T. Kanda was supported by the Kajima Corporation, Tokyo, Japan. Special thanks are due to J. Hikasa from Kuraray Co., Ltd., Osaka, Japan, for helpful discussions and material supplies. Dr. H. C. Wu at the University of Michigan is acknowledged for his valuable advice.

\section{APPENDIX III. REFERENCES}

Akers, S. A. S., et al. (1989). "Long term durability of PVA reinforcing fibers in a cement matrix." Int. J. Cement Compos. and Lightweight Concrete, 11, 79-91.

Akihama, S., Suenaga, T., Nakagawa, H., and Suzuki, K. (1985). "Experimental study of vinylon fiber reinforced cement composites "VFRC" (Part I)." Kajima Annu. Tech. Rep., Vol. 33, Kajima Corp., Tokyo, Japan, 123-128 (in Japanese).

Akihama, S., Suenaga, T., and Sakano, T. (1982). "Experimental study of carbon fiber reinforced cement composites "CFRC" (Part II). Kajima Annu. Tech. Rep., Vol. 3, Kajima Corp., Tokyo, Japan, 123-128 (in Japanese).

Betterman, L. R., Ouyang, C., and Shah, S. P. (1995). "Fiber-matrix interaction in microfiber-reinforced mortar." J. Advanced Cement Based Mat., 2, 53-61.

Chan, Y. W., and Li, V. C. (1997a). "Age effect on the characteristics of fiber/cement interfacial bond." J. Mat. Sci., 32(19), 5287-5292.

Chan, Y. W., and Li, V. C. (1997b). "Effects of transition zone densification on fiber/cement paste bond strength improvement." J. Advanced Cement Based Mat., 5(1), 8-17.

Gao, Y., Mai, Y. W., and Cotterell, B. (1988). "Fracture of fiber-reinforced materials." J. Appl. Mathematics and Physics (ZAMP), 39(4), 550572.

Goplaratnam, V. S., and Shah, S. P. (1985). "Tensile fracture of steel fiber reinforced concrete." J. Engrg. Mech., ASCE, 113(4), 635-653.

Hirsch, T. J. (1962) "Modulus of elasticity of concrete affected by elastic moduli of cement paste matrix and aggregate." J. Am. Concrete Inst., March, 427-449.

Katz, A., and Li, V. C. (1996). "A special technique for determining the bond strength of carbon fibers in cement matrix by pullout test." $J$. Mat. Sci. Letters, 15, 1821-1823.

Kozey, V. V., Jiang, H., Mehta, V. R., and Kumar, S. (1995). "Compressive behavior of materials: part II. High performance fibers." J. Mat. Res., 10(4), 1044-1061.

Leung, C. K. Y. (1992). "Fracture-based two-way debonding model for discontinuous fibers in elastic matrix." J. Engrg. Mech., ASCE, 118(11), 2298-2318.

Leung, C. K. Y., and Li, V. C. (1991). "New strength-based model for the debonding of discontinuous fibers in an elastic matrix." J. Mat. Sci., 26(11), 5996-6010.

Li, V. C., and Chan, Y. W. (1995). "Determination of interfacial debonding mode for fiber-reinforced cementitious composites." J. Engrg. Mech., 120(4), 707-719.

Li, V. C., and Leung, K. Y. (1992). "Steady-state and multiple cracking of short random fiber composites." J. Engrg. Mech., ASCE, 118(11), $2246-2263$.

$\mathrm{Li}$, V. C., and Stang, H. (1997). "Interface property characterization and strengthening mechanisms in fiber reinforced cement based composites." J. Advanced Cement Based Compos., J. Advanced Cement Based Mat., 6(1), 1-20.

Li, V. C., Wang, Y., and Backer, S. (1990). "Effect of inclining angle, bundling, and surface treatment on synthetic fiber pull-out from a cement matrix." Composites, 21(2), 132-140.

Li, V. C., Wang, Y., and Backer, S. (1988). "Analysis of synthetic fiber pull-out from a cement matrix." Bonding in cementitious composites, S. Mindess and S. Shah, eds., Vol. 114, Mat. Res. Soc., 159-166.

Li, V. C., Wu, H. C., and Chan, Y. W. (1996). "Effect of plasma treatment of polyethylene fibers on interface and cementitious composite properties." J. Am. Ceramic Soc., 79(3), 700-704.

Maalej, M., Li, V. C., and Hashida, T. (1995). "Effect of fiber rupture on tensile properties of short fiber composites." J. Engrg. Mech., ASCE, 121(8), 903-913.

Morrison, J. K., Shah, S. P., and Jenq, Y. S. (1988). "Analysis of fiber debonding and pullout in composites." J. Engrg. Mech., ASCE, 114(2), 277-294.

Naaman, A. E., Namur, G. C., Alwan, J. M., and Najm, H. (1991). "Fiber pullout and bond slip I (analytical study)." J. Engrg. Mech., ASCE, 117(7), 2769-2790.

Piggott, M. R. (1974). "Toughness in obliquely-stressed fibrous composites." J. Mech. Phys. Solids, 22, 457-468.

"PVA fiber as reinforcing materials for cement." (1986). Kuraray Corp. Tech. Rep., Osaka, Japan (in Japanese).

Stang, H., and Shah, S. P. (1986). "Failure of fiber reinforced composites by pull-out fracture." J. Mat. Sci., 21(3), 953-958.

Stang, H., Li, Z., and Shah, S. P. (1990). "The pull-out problem; the stress versus fracture mechanics approach.” J. Engrg. Mech., ASCE, $116(10), 2135-2150$.

\section{APPENDIX IV. NOTATION}

The following symbols are used in this paper:

$d_{f}=$ fiber diameter;

$E_{c}=$ elastic modulus of composite;

$E_{f}=$ elastic modulus of fiber;

$E_{m}=$ elastic modulus of matrix;

$G_{c}=$ shear modulus of composite;

$L_{d}=$ fiber debonding length;

$L_{f}=$ fiber length;

$l_{\text {crit }}=$ critical embedment length of fiber;

$l_{e}=$ fiber embedment length;

$P_{a}=$ peak load of single fiber pullout curve;

$P_{b}=$ load after sudden drop following $P_{a}$;

$R^{*}=$ effective radius of matrix cylinder containing fiber;

$V_{m}=$ volume fraction of matrix;

$\delta$ = fiber-end displacement;

$\sigma_{d}=$ fiber stress;

$\sigma_{f u}=$ apparent fiber strength;

$\sigma_{f u}^{n}=$ nominal tensile strength of fiber (embedded fiber strength at $\phi=0)$;

$\tau_{i}=$ frictional bond strength;

$\tau_{s}=$ chemical bond strength; and

$\phi=$ fiber inclining angle. 